\title{
Reversible Posterior Leucoencephalopathy Syndrome Following Duloxetine: A Case Report and Review of Literature
}

\author{
Christine Chhakchhuak ${ }^{\mathrm{a}, \mathrm{b}}$, Mohammad Anower ${ }^{\mathrm{a}}$, Mukta Panda ${ }^{\mathrm{a}}$
}

\begin{abstract}
Reversible Posterior Leucoencephalopathy Syndrome (RPLS) is a disorder characterized by headaches, confusion, and/or decreased level of consciousness, seizures, and visual changes. A 58-year-old female was found unresponsive, with generalized rigidity and urinary incontinence for unknown duration. On presentation, she was febrile, tachycardic, tachypneic, and hypertensive. Neurological exam showed increased muscle tone and brisk deep tendon reflexes. Brain MRI revealed gyriform edema and white matter hypodensities in parieto-occipital regions consistent with RPLS. Discontinuation of duloxetine, one of her home medications, led to resolution of symptoms. We report this case to discuss the syndrome of RPLS and propose the role of duloxetine in causing hypertension and cerebral vasoconstriction leading to RPLS. To our knowledge, this is the first reported case of RPLS resulting from the use of duloxetine.
\end{abstract}

Keywords: Reversible posterior leucoencephalopathy syndrome; Duloxetine; Cerebral vasoconstriction; Adverse reactions

\section{Introduction}

Reversible posterior leucoencephalopathy syndrome (RPLS) as a distinct entity was first described in 1996 by Hinchey et al. This disorder is characterized primarily by headaches, confusion/decreased level of consciousness, seizures, and visual changes [1]. RPLS, as we know today, is a clinical

Manuscript accepted for publication November 18, 2010

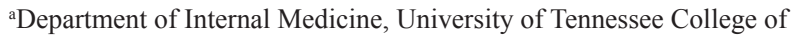
Medicine, Chattanooga, TN, 37403, USA

${ }^{b}$ Corresponding author: University of Tennessee College of Medicine, 960 East Third Street Suite 200 Chattanooga, TN 37403, USA

Email: chris_chkchk@yahoo.co.in

doi:10.4021/jmc98w radiologic syndrome linked to a wide variety of etiologies. By far the most common etiology linked to the pathogenesis of RPLS is hypertension $[2,3]$. Other factors implicated in the development of RPLS include eclampsia [4], immunosuppressive medications $[1,5]$, and a variety of other drugs [5-7]. The pathogenesis of RPLS remains controversial but mainly involves loss of cerebral autoregulation and endothelial dysfunction [1]. Diagnosis is established by neuroimaging. Typical findings are symmetrical white matter edema in the posterior cerebral hemispheres, particularly the parietooccipital regions [8]. Treatment of RPLS includes rapid lowering of blood pressure, antiepileptics, and withdrawal of offending agent $[9,10]$.

In our case, we discuss the syndrome of RPLS and we propose the possible role of duloxetine in causing hypertension and cerebral vasoconstriction leading to RPLS. To our knowledge, there has been no reported case of RPLS secondary to the use of duloxetine in the English literature.

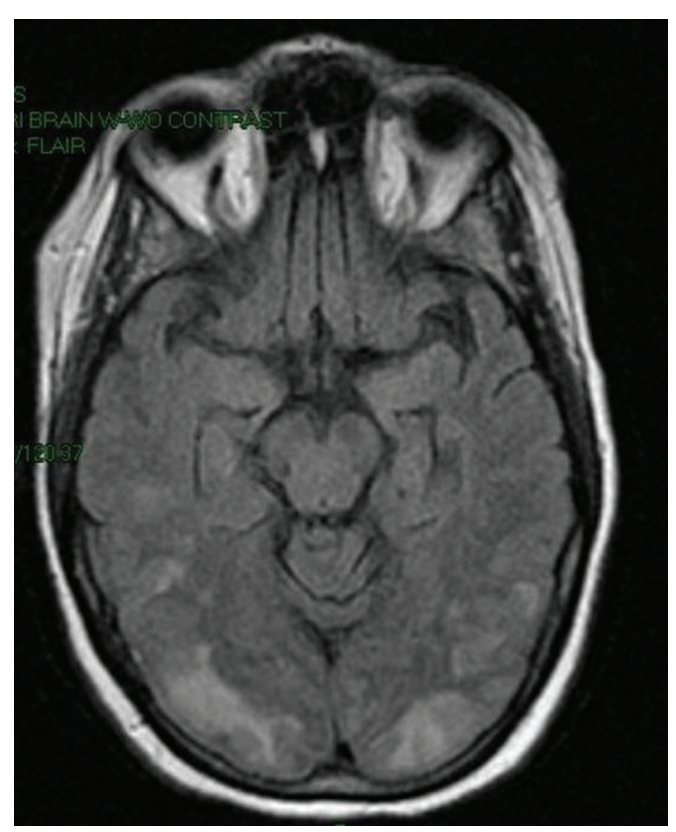

Figure 1. MRI image of the brain showing gyriform edema and white matter hypodensities in parieto-occipital regions. 


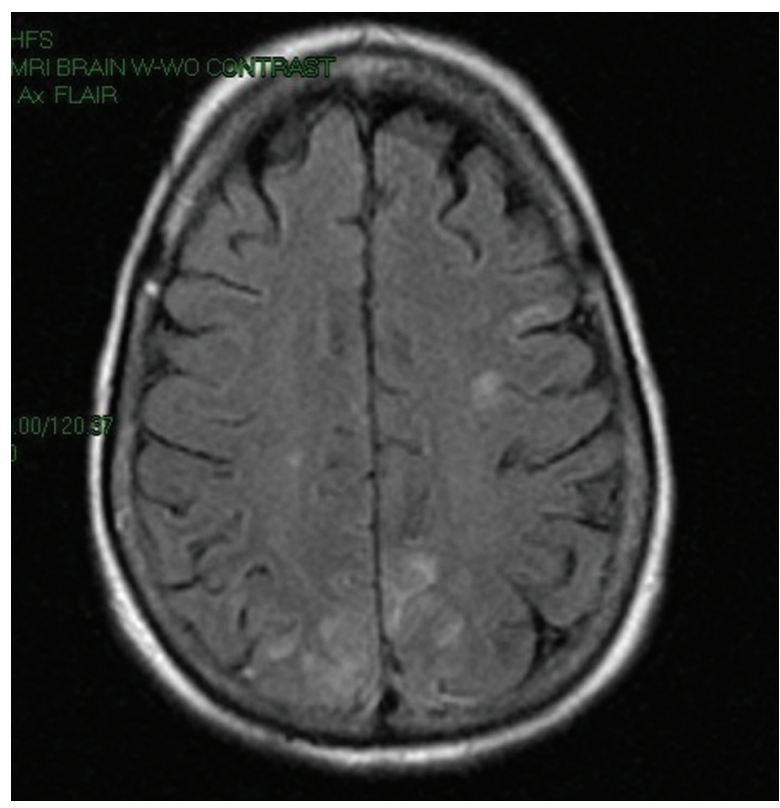

Figure 2. MRI image of the brain showing gyriform edema and white matter hypodensities in parieto-occipital regions.

\section{Case Report}

A 58-year-old Caucasian female was brought to the emergency room by Emergency Medical Services (EMS) after being found with generalized body rigidity, urinary incontinence, and unresponsiveness for an unknown duration of time. The patient was intubated for airway protection by the EMS personnel. She was last seen normal the day prior to admission. Her past medical history was significant for hypertension, chronic pain syndrome, depression, and hypothyroidism. She had attempted suicide (overdose of antidepressants) twice in the past. Her home medications included duloxetine, zolpidem, cyclobenzaprine, pregabalin, oxycodone, amlodipine, and benazepril. She was working as a nurse at a psychiatric institute. There was no history of smoking, alcohol abuse, or drug abuse. At the time of initial presentation, her vital signs were blood pressure 220/126 $\mathrm{mmHg}$, pulse 135/min, respiratory rate 42/min, temperature 103 degrees Fahrenheit and $\mathrm{O} 2$ saturation $97 \%$ on the ventilator. Physical exam revealed a well nourished, disheveled Caucasian lady who was intubated. Neurological exam was significant for bilaterally symmetric and reactive pupils, positive gag reflex, spontaneous movement in all extremities, increased muscle tone, brisk and symmetric deep tendon reflexes, downgoing bilateral plantar reflexes, and no signs of meningism or clonus. Higher mental functions could not be assessed as she was sedated. Rest of the systemic exam was normal. Initial laboratory data showed mild leucocytosis, normal complete metabolic panel, and mildly elevated troponins and CPK. Based on the initial presentation and concern for possible menin- goencephalitis, the patient was started on a broad spectrum antibiotic coverage with vancomycin, rocephin, and ampicillin. Blood cultures, urine culture, and CSF gram stain and culture were obtained prior to initiation of antibiotics. On the second day of hospitalization, acyclovir was also added to the regimen for possible herpes simplex encephalitis. During the rest of the hospital course, the patient's cultures came back sterile, herpes simplex PCR was negative and there was no growth of organisms from the CSF as well. EEG done on the patient showed a generalized slowing consistent with an encephalopathy. CT of the head done without contrast was unremarkable. MRI of the head revealed gyriform edema and white matter hypodensities in parieto-occipital regions (Fig. 1, 2). Considering the negative infective etiology and the MRI findings the diagnosis of RPLS was considered. Antibiotics were discontinued and supportive care was provided to the patient. The patient's home medications that had been continued in hospital were reviewed again and duloxetine was discontinued, which led to complete resolution of symptoms within 5 days. The patient was discharged home to be followed up as an outpatient. Subsequently, the patient has had follow-up neuroimaging 6 weeks post her discharge with complete resolution of the initial MRI findings (Fig. 3, 4).

\section{Discussion}

Reversible Posterior Leucoencephalopathy Syndrome (RPLS) is a clinical radiologic syndrome that was first described as a separate entity in 1996 [1]. Some other names used for this syndrome include Posterior Reversible Encephalopathy Syndrome (PRES), hyperperfusion encephalopathy, and brain capillary leak syndrome.

RPLS is becoming an increasingly recognized neurologic disorder. The exact mechanism by which this condition is

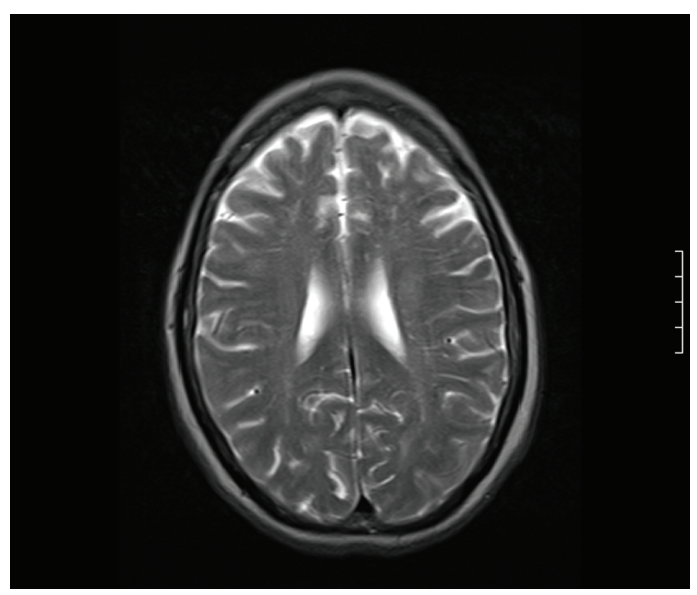

Figure 3. MRI Brain 6 weeks later showing complete resolution of the parieto-occipital changes. 


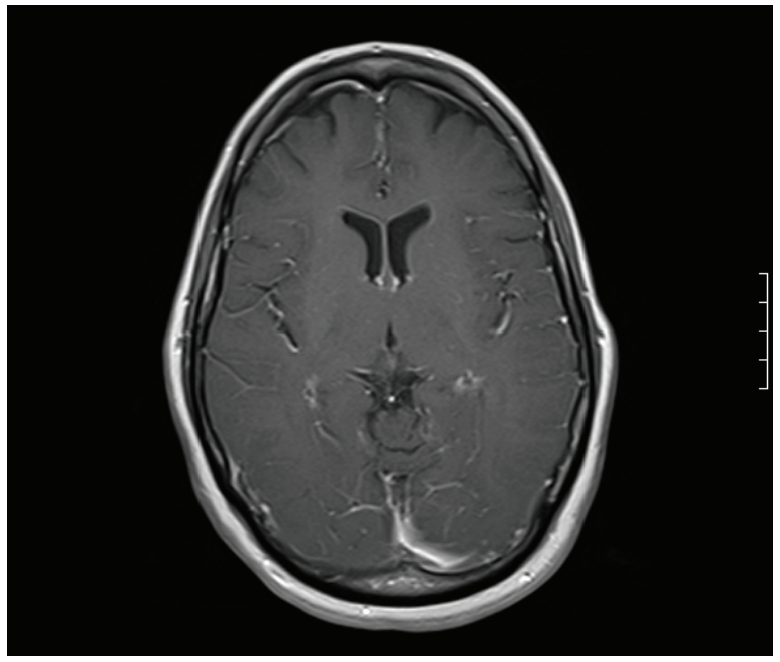

Figure 4. MRI Brain 6 weeks later showing complete resolution of the parieto-occipital changes.

caused is not known though there have been two postulated theories, the cytotoxic theory and the vasogenic theory. According to the cytotoxic theory, a direct toxic effect on the endothelial cells of the cerebral vasculature results in cell autonomous dysfunction. Whereas, according to the vasogenic theory, an acute increase in blood pressure overrides the autoregulation of cerebral mean arterial pressure, leading to vasodilatation and autonomous endothelial insult $[9,11]$. The end result via both pathways is the diffusion of plasma proteins and cells into the extracellular space and subsequent cerebral edema.

Various precipitating factors have been identified. Hypertension is believed to be the most common precipitant in most cases [3]. However, RPLS can also occur in the absence of hypertension. Clinical conditions that have been associated with RPLS are acute glomerulonephritis, preeclampsia and eclampsia, systemic lupus erythematosus, thrombotic thrombocytopenic purpura, and hemolytic-uremic syndrome [12]. Immunosuppressive medications and a variety of other drugs have been implicated in the development of RPLS [57].

Selective Serotonin Reuptake Inhibitors (SSRIs) can uncommonly cause a syndrome known as reversible cerebral vasoconstriction syndrome (RCVS). This is a syndrome characterized by the association of severe headaches with or without additional neurological symptoms and a 'string of beads' appearance on cerebral arteries, which resolve spontaneously in 1-3 months. RPLS is amongst the early complications of reversible cerebral vasoconstriction syndrome and usually occurs within the first week [13]. Ducros et al. reported a case series of 67 patients with RCVS, of which RPLS was reported as a complication in $9 \%$ of these cases and SSRIs were implicated in $21 \%$ of the cases of RCVS [14]. Paroxetine, citalopram, fluoxetine, and sertraline are among the SSRIs reported to be linked to RCVS in this case series. Duloxetine, although has a very similar pharmacological profile as the other SSRIs, differs from the class in being a potent reuptake inhibitor of norepinephrine as well [15]. We believe in our case, the use of duloxetine resulted in serotonin syndrome which led to the sequential development of hypertension, RCVS, and finally RPLS. To our knowledge, there has been no evidence in the literature to suggest the role of duloxetine in causing RCVS or RPLS.

The presenting clinical features of RPLS include headaches, seizures, visual disturbances including cortical blindness and visual hallucinations, and alterations in mental status including confusion and stupor $[1,9]$. The headache can range from moderate to severe and may not respond to analgesics [9]. Seizures can be the initial presenting complaint as was the case in our patient as well [1]. Seizures are usually of the tonic-clonic type. Hypertension is accompanied with the neurologic symptoms most of the time and may precede the symptoms by a few hours [9].

The diagnosis of RPLS is established by neuroimaging. Typical findings are symmetrical white matter edema in the posterior cerebral hemispheres, particularly the parietooccipital regions [8]. Involvement of cerebellum and brain stem is common. The calcarine and paramedian parts of the occipital lobe are usually spared which might help in differentiating from bilateral posterior cerebral infarctions [1]. Although depicted well on CT, the findings are best seen on MRI. The most common abnormalities seen on MRI are confluent areas of increased signal on proton density and T2weighted images [8].

Early diagnosis and treatment is essential for the patients' prognosis. Discontinuation of the offending agent and control of blood pressure are pivotal to reverse the fatal consequences [13]. Hypertension is a frequent feature in cases of RPLS and patients can improve dramatically with lowering of blood pressure [9]. Most seizures respond well to phenytoin and the drug can be tapered off quickly with reversal of neurological symptoms and imaging findings [9]. The prognosis of RPLS is good and symptoms are reversible if diagnosis is made promptly and remedial measures are taken.

\section{References}

1. Hinchey J, Chaves C, Appignani B, Breen J, Pao L, Wang A, Pessin MS, et al. A reversible posterior leukoencephalopathy syndrome. N Engl J Med 1996;334(8):494-500.

2. Schwartz RB, Jones KM, Kalina P, Bajakian RL, Mantello MT, Garada B, Holman BL. Hypertensive encephalopathy: findings on CT, MR imaging, and SPECT imaging in 14 cases. AJR Am J Roentgenol 1992;159(2):379-383.

3. Schwartz RB, Mulkern RV, Gudbjartsson H, Jolesz F. 
Diffusion-weighted MR imaging in hypertensive encephalopathy: clues to pathogenesis. AJNR Am J Neuroradiol 1998;19(5):859-862.

4. Raps EC, Galetta SL, Broderick M, Atlas SW. Delayed peripartum vasculopathy: cerebral eclampsia revisited. Ann Neurol 1993;33(2):222-225.

5. Rajasekhar A, George TJ, Jr. Gemcitabine-induced reversible posterior leukoencephalopathy syndrome: a case report and review of the literature. Oncologist 2007; 12(11):1332-1335.

6. Eran A, Barak M. Posterior reversible encephalopathy syndrome after combined general and spinal anesthesia with intrathecal morphine. Anesth Analg 2009;108(2):609-612.

7. Cumurciuc R, Martinez-Almoyna L, Henry C, Husson $\mathrm{H}$, de Broucker T. Posterior reversible encephalopathy syndrome during sunitinib therapy. Rev Neurol (Paris) 2008;164(6-7):605-607.

8. Lamy C, Oppenheim C, Meder JF, Mas JL. Neuroimaging in posterior reversible encephalopathy syndrome. $\mathrm{J}$ Neuroimaging 2004;14(2):89-96.

9. Stott VL, Hurrell MA, Anderson TJ. Reversible pos- terior leukoencephalopathy syndrome: a misnomer reviewed. Intern Med J 2005;35(2):83-90.

10. Hauben M. Cyclosporine neurotoxicity. Pharmacotherapy 1996;16(4):576-583.

11. Kwon S, Koo J, Lee S. Clinical spectrum of reversible posterior leukoencephalopathy syndrome. Pediatr Neurol 2001;24(5):361-364.

12. Calabrese LH, Dodick DW, Schwedt TJ, Singhal AB. Narrative review: reversible cerebral vasoconstriction syndromes. Ann Intern Med 2007;146(1):34-44.

13. Gijtenbeek JM, van den Bent MJ, Vecht CJ. Cyclosporine neurotoxicity: a review. J Neurol 1999;246(5):339346.

14. Ducros A, Boukobza M, Porcher R, Sarov M, Valade $\mathrm{D}$, Bousser MG. The clinical and radiological spectrum of reversible cerebral vasoconstriction syndrome. A prospective series of 67 patients. Brain 2007;130(Pt 12):3091-3101.

15. Westanmo AD, Gayken J, Haight R. Duloxetine: a balanced and selective norepinephrine- and serotonin-reuptake inhibitor. Am J Health Syst Pharm 2005;62(23):2481-2490. 